\title{
Interaction of new butyltin citrate complex with lipid model membrane and DNA
}

\author{
Hanna Pruchnik Teresa Kral $\cdot$ Martin Hof
}

Received: 1 November 2013/Accepted: 28 March 2014/Published online: 22 April 2014

(C) The Author(s) 2014. This article is published with open access at Springerlink.com

\begin{abstract}
Organotin(IV) complexes show a wide variety of biological activity: bactericidal, fungicidal, caricidal, and pesticidal. Many of them are more effective than traditional heavy metal anticancer drugs. The aim of the present study was to investigate the interaction of a new dibutyltin complex with citric acid (DBTC) with lipid membrane and plasmid DNA. The effect of this compound on the multilamellar liposomes formed with dipalmitoylphosphatidylcholine, dimyristoylphosphatidylcholine, and dimyristoylphosphatidylethanolamine was studied mainly by the means of differential scanning calorimetry and additionally by the steady-state fluorimetry. Calorimetrical results together with fluorescence spectroscopy suggest that investigated complex interacts with lipids and probably locates itself in the hydrophilic part of the membrane. The effect of DBCT on plasmid DNA was investigated using single-molecule florescence technique and time-correlated single photon counting fluorescence correlation spectroscopy. We could conclude then that DBTC-DNA interaction occurs due to the interaction with the DNA phosphate group and charge neutralization which then leads to DNA compaction.
\end{abstract}

Keywords Butyltin citrate complex · Phase transition . DSC $\cdot$ Lipid membrane $\cdot$ DNA $\cdot$ TCSPC-FCS

H. Pruchnik $(\bowtie) \cdot$ T. Kral

Department of Physics and Biophysics, Wrocław University of Environmental and Life Sciences, Norwida 25, 50-375 Wrocław, Poland

e-mail: hanna.pruchnik@up.wroc.pl

T. Kral · M. Hof

J. Heyrovsky Institute of Physical Chemistry of the ASCR, v.v.i., Dolejškova 2155/3, 18223 Prague 8, Czech Republic

\section{Introduction}

Organotin(IV) complexes play an important role in medicine, agriculture, and industry. The compounds show a wide variety of biological activity: bactericidal, fungicidal, caricidal, and pesticidal [1-6]. In recent years, many triorgano- and diorganotin compounds have been synthesized and tested for their antitumor activity in vitro against a large variety of tumor lines. Many of them are more effective than traditional heavy metal anticancer drugs $[3,6]$. A series of triorgano- and diorganotin carboxylates, aminocarboxylates, oxamates etc., display interesting antitumor activity. Biological activity of organotin depends not only on the number and nature of $\mathrm{Sn}-\mathrm{C}$ bonds but also on the mode of coordination of ligands and their electronic properties. The most active antitumor agents are diorganotin and triorganotin compounds [1-9]. Antitumor activity depends on the properties of group coordinated to tin atom and most frequently diminishes in the order: $n-\mathrm{Bu}>\mathrm{Ph}>n-\mathrm{Pr}>\mathrm{Et}>\mathrm{Me}[2-8,10-12]$. The mechanism of biological action of organotin derivatives is still not clear but due to their lipophilicity organotins are membrane-active-for example, disruption of membrane integrity may occur because of organotin binding or insertion into the membrane. Organotins can also act intracellulary disrupting intact organelles [3]. The organotin compounds may interact with DNA, membrane proteins, glycoproteins, and cellular proteins. Some authors proposed that DNA is the probable target for the cytotoxic activity of organotin complex [4, 9]. The binding ability of these complexes with DNA depends on the coordination number and nature of the groups bonded to the central tin atom, e.g., the authors [11] suggest that the ligand of organotin(IV) carboxylate complexes binds with DNA double helix by hydrogen bonding between the ligand and 


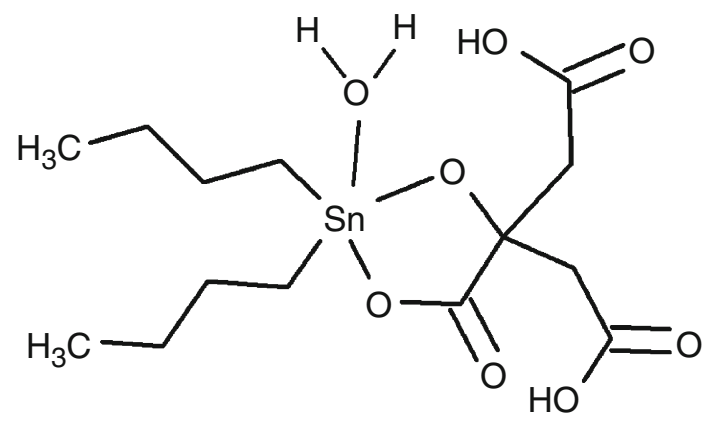

Fig. 1 Chemical structure of dibutyltin citrate complex in solution

the base pairs in DNA typically to $\mathrm{N}_{3}$ of adenine and $\mathrm{O}_{2}$ of thymine as well as partial intercalation of aromatic ring into the base pairs of DNA. The interaction of organotin compounds with DNA is of interest for therapeutic treatment as these molecules recognize specific DNA sequences, alter the local DNA structure, inhibit access to the activator or repressor protein, and ultimately affect the gene-expression process [13].

The subject of the research is the new dibutyltin complex with citric acid- $\left[\mathrm{Sn}\left(\mathrm{C}_{4} \mathrm{H}_{9}\right)_{2}\left(\mathrm{C}_{6} \mathrm{H}_{6} \mathrm{O}_{7}\right)\right]-$ DBTC (Fig. 1). The organotin compounds with citrato acid have been investigated to a lesser extent than other organotin carboxylates. However, they play important role in organotin biochemistry because of their relatively large concentrations in the environment and high biological activity [14]. In the previous paper, we examined properties of dibutyltin complex with citric acid and its interaction with nucleotides. The NMR spectroscopy showed that DBTC effectively interact with disodium 2'-deoxyadenosine $5^{\prime}$-monophosphate (DAMP), adenosine $5^{\prime}$-monophosphate monohydrate (AMP), and adenosine $5^{\prime}$-triphosphate disodium salt (ATP). The Sn atoms of DBTC are coordinated to chelating carboxylate ligands and nucleotide molecules via phosphate groups; however, coordination of nucleotide to $\mathrm{Sn}$ atoms as a chelating ligand via phosphate group and $\mathrm{N}_{7}$ atom of adenine residue is also possible [15]. Our preliminary investigation shows that the dibutyltin citrate complex is effective cytotoxic agent against Caco-2 and A549 tumor cells (Pruchnik et al., paper in preparation).

The aim of this paper is to present the study of interactions of the new butyltin citrate complex with lipid membranes and plasmid DNA. In particular, its ability to compact (binding) DNA and its influence on the membrane structure and thermotropic phase behavior was studied. The effect of the DBTC on the lipid bilayer phase transition was examined mainly by differential scanning calorimetry (DSC) and additionally by fluorescence spectroscopy. The above mentioned methods have been employed to systematically monitor the influence of the CGA on different membrane regions. The degree of packing order of the hydrophilic phase of the lipid bilayer was determined by the Laurdan and Prodan fluorescence probes, while the fluorescence anisotropy of the hydrophobic phase-with the DPH probe. DSC is a useful method in studies on the nature of biological systems such as lipids or proteins [16-18]. Thermal parameters that were monitored include the temperature at the pretransition $\left(T_{\mathrm{p}}\right)$ and the main transition peak $\left(T_{\mathrm{m}}\right)$, the half width $\left(\Delta T_{1 / 2}\right)$ and change of the enthalpy $(\Delta H)$ of the main transition. To understand the interaction of butyltin citrate complex with plasmid DNA, we used a single-molecule florescence technique and timecorrelated single photon counting fluorescence correlation spectroscopy (TCSPC-FCS).

\section{Materials and methods}

\section{Chemicals}

The compound dibutyltin complex with citric acid$\left[\mathrm{Sn}\left(\mathrm{C}_{4} \mathrm{H}_{9}\right)_{2}\left(\mathrm{C}_{6} \mathrm{H}_{6} \mathrm{O}_{7}\right)\right]$ was prepared by procedures reported earlier [15]. Numerical calculations reported in the same paper suggested that the most probable structure of this compound is as shown on Fig. 1.

The 1,2-dipalmitoyl-sn-glycero-3-phosphatidylcholine (DPPC), 1,2-dimirystoyl-sn-glycero-3-phosphatidylcholine (DMPC), and 1,2-dimirystoyl-sn-glycero-3-phosphatidyletanolamine (DMPE) lipids were purchased from Sigma Aldrich, Steinheim, Germany. The fluorescent probes: 6-dodecanoyl-2-dimethylaminonaphthalene (Laurdan), 6-propionyl-2-dimethylaminonaphthalene (Prodan), and 1,6-diphenyl-1,3,5-hexatriene (DPH) were purchased from Molecular Probes, Eugene, OR, USA.

The 10,000 bp (Bap) plasmid and two fluorescent dyes PicoGreen $^{\circledR}$ (PG) and Hoechst $33258^{\circledR}$ (H33258) (Molecular Probes) were prepared and used as described elsewhere (M3). We used the labeling ratio 2 dye molecules/ 100 base pairs. After staining, DNA plasmid was titrated with various amounts of DBTC. Experiments were performed in deionized water at $25^{\circ} \mathrm{C}$.

\section{Differential scanning calorimetry}

Samples for DSC were prepared from multilamellar liposomes (MLV) of phosphatidylcholine (DPPC, DMPC) and phosphatidyloetanolamine (DMPE). The lipids and compound were dissolved in chloroform and in ethanol, respectively. Chloroform and ethanol were very carefully evaporated to dryness under nitrogen and thin film was formed on the flask wall, after which distilled water was added. The lipid film was dispersed by agitating the flask on a vortex mixer to give a milky suspension of liposomes 
at a temperature above the main phase transition of phosphatidylcholine. Final lipids' concentration in the samples was $25 \mathrm{mg} \mathrm{cm}^{-3}$. The prepared MLV of lecithin and lecithin with the compounds added were encapsulated in $40 \mu \mathrm{l}$ volumes and incubated for 4 days at $4{ }^{\circ} \mathrm{C}$. The measurements were made with the Mettler Toledo Thermal Analysis System D.S.C. $821^{\mathrm{e}}$ operated at a heating rate of $2{ }^{\circ} \mathrm{C} \mathrm{min}{ }^{-1}$ from 20 to $55^{\circ} \mathrm{C}$ of DPPC, from 10 to $35^{\circ} \mathrm{C}$ of DMPC, and from 20 to $60{ }^{\circ} \mathrm{C}$ of DMPE. Thermal cycles were repeated three times, the experimental error in temperature and thermal response were $\pm 0.2{ }^{\circ} \mathrm{C}$ and $\pm 5 \%$, respectively. Data analysis was done using original software provided by Mettler Toledo in order to determine the temperature of the pre- $\left(T_{\mathrm{p}}\right)$ and main transition $\left(T_{\mathrm{m}}\right)$, the main half-width transition $\left(\Delta T_{1 / 2}\right)$, and the calorimetric enthalpy $(\Delta H)$.

\section{Fluorescence spectroscopy}

Samples for steady-state fluorimetry consisted of MLVs with DPPC and DMPC all modified with dibutyltin complex (DPPC-DBTC, DMPC-DBTC). The MLV were prepared identically as for DSC measurements. The lipids, compound, and fluorescence probes (Laurdan, Prodan and $\mathrm{DPH}$ ) were dissolved in chloroform and in ethanol, respectively. Chloroform and ethanol were very carefully evaporated to dryness under nitrogen and thin film was formed on the flask wall, after which distilled water was added. The lipid film was dispersed by agitating the flask on a vortex mixer to give a milky suspension of liposomes at a temperature above the main phase transition of phosphatidylcholine. Samples were incubated $30 \mathrm{~min}$ in darkness at room temperature. Final lipids' concentration in the samples was $450 \mu \mathrm{M}$, molar ratio of citric complex and lipids was 0.07 , and concentration of fluorescence probes was $10 \mu \mathrm{M}$. The measurements were made at different temperatures - above and below the main phase transition of DPPC or DMPC. Thermal cycles $\left(20-60\right.$ or $10-50{ }^{\circ} \mathrm{C}$ ) were repeated three times.

The measurements were conducted with a CARY Eclipse of VARIAN fluorimeter equipped with a Peltier temperature controller DBS (temp. accuracy $\pm 0.1^{\circ} \mathrm{C}$ ). The excitation and emission wavelengths for DPH probe were $\lambda_{\mathrm{ex}}=360 \mathrm{~nm}, \lambda_{\mathrm{em}}=425 \mathrm{~nm}$. The excitation wavelength for Laurdan and Prodan was $360 \mathrm{~nm}$, and the emitted fluorescence was recorded at two wavelengths: 440 and $490 \mathrm{~nm}$.

Fluorescence anisotropy $(A)$ for DPH probes was calculated using the formula $A=\left(I_{\mathrm{II}}-G I_{\perp}\right) /\left(I_{\mathrm{II}}+G I_{\perp}\right)$, where $I_{\mathrm{II}}$ and $I_{\perp}$-fluorescence intensities observed in directions parallel and perpendicular to the polarization direction of the exciting wave. $G$ is an apparatus constant dependent on the emission wavelength [20]. Changes in the polar group packing arrangement of the hydrophilic part of the membrane were investigated using Laurdan and Prodan probes, on the basis of generalized polarization (GP), and were calculated with the formula GP $=\left(I_{\mathrm{g}}-I_{1}\right) /\left(I_{\mathrm{g}}+I_{1}\right)$, where $I_{\mathrm{g}}$ and $I_{1}$ are the fluorescence intensities at the gel and the fluid phase, respectively [19, 20].

Time-correlated single photon counting fluorescence correlation spectroscopy (TCSPC-FCS)

To explore the dibutyltin citrate complex-DNA interactions, a single-molecule detection technique that combines simultaneous time-correlated single photon counting time tag mode with fluorescence correlation spectroscopy (TCSPC-FCS) was employed, as reported earlier [21-23] Mainly, there are two readout parameters the residence time $\left(\tau_{\text {res }}\right)$ and apparent particle number $(\mathrm{PN})$ contain information about the diffusion coefficient and the concentration of the fluorescently labeled molecules, respectively [24]. $\mathrm{PN}$ is described by the equation $\mathrm{PN}=C \times V \times N_{\mathrm{A}}$, where $C$ is molarity of detected molecules (DNA), $V$ is confocal volume ( $1 \mathrm{fL}$ ), and $N_{\mathrm{A}}$ is the Avogadro constant. The DNA concentration used in experiments was constant at $1 \mathrm{nM}$, the theoretical PN to be achieved is around 0.6. However, in the case reported herein the multi-labeled plasmid DNA might exceed the diameter of the laser focus about one and half times [25, 26]. For the freely diffusing plasmid DNA, the segmental and the Brownian motions of the whole molecule are superimposed [27], which causes an increase of the apparent number of particles in the focus and also affects the apparent residence times. The combination of these FCS measurements with the TCSPC method allows simultaneous detection of the fluorescence lifetime. The fluorescence lifetime gives information on the local environment both PicoGreen and Hoechst label. As shown earlier [23, 28], it can be interpreted as a mark of DNA tight compaction.

The set-up was as follows: TCSPC-FCS measurements were performed on a MicroTime 200 inverted confocal microscope (PicoQuant, Germany) with the pulsed diode laser (LDH-P-C-470, $470 \mathrm{~nm}$ PicoQuant) providing $80 \mathrm{ps}$ pulses at a $40 \mathrm{MHz}$ repetition rate, dichroic mirror 490 DRLP and band-pass filter 515/50 (Omega Optical), and a water immersion objective (1.2 NA, 60×) (Olympus). Low power of $4 \mu \mathrm{W}$ (at the back aperture of the objective) was chosen to minimize photobleaching and saturation. In the detection plane, a pinhole ( $50 \mu \mathrm{m}$ in diameter) was used and the signal was collected by a single photon avalanche diode (SPAD, Microphoton Devices, Bolzano, Italy). Photon arrival times were stored using fast electronics (Picoharp 300, PicoQuant) in time-tagged time-resolved recording mode. Two independent times were assigned to each detected photon: (i) a time after the beginning of the measurement and (ii) a time after the previous laser pulse. 
The FCS data analysis was done using home-built routines $($ DevC ++ , Bloodshed Software and OriginPro70, OriginLab Corporation). The TCSPC data analysis was performed using SymPhoTime software (PicoQuant). Further details of the data evaluation are given elsewhere [27].

\section{Results and discussion}

Interaction with lipid membrane

Interaction of dibutyltin citrate complex with lipid membranes was investigated by the means of DSC and fluorescence spectroscopy. The molecular models used in our studies of interaction of DBTC with membranes consist of bilayers of DPPC, DMPC, and DMPE which are representative of phospholipid classes located in the outer and inner monolayers of many cells including those of the human erythrocyte membrane. Using calorimetry, we also examined the heat signal associated: (1) with the lamellar gel phase to ripple phase and (2) with the ripple phase to fluid lamellar phase transition of MLV of phosphatidylcholine. The effect of DBTC on the phase transition of DPPC determined in the DSC experiment is shown in Fig. 2. In the absence of the compound, two characteristic endothermic peaks are visible referring to pretransition (1) and main transition (2), respectively. The presence of DBTC reduces cooperativity of the main phase transition and for increasing concentration abolished the pretransition. Below a molar ratio of 0.03 , dibutyltin complex practically does not affect the main phase transition temperature $\left(T_{\mathrm{m}}\right)$ and the $T_{\mathrm{p}}$, but shows effect on the transition enthalpy (Fig. 4). For a molar ratio of 0.07 and above DBTC abolished the pretransition and scarcely affects the $T_{\mathrm{m}}$ and enthalpy. The main phase transition is broadened and asymmetrical, with visible shoulders. Since the pretransition is a result of changes in acyl chain packing, its perturbation and/or vanishing indicates that DBTC probably influences the structure of the lipid bilayer. On the other hand, compounds that incorporate into the polarnonpolar region of the lipid bilayer abolish the DPPC pretransition even at low concentrations [29]. At the higher concentration ( 0.1 and 0.2 molar ratio), the main phase transition peak splits into two components: the first corresponding to the $T_{\mathrm{m}}$ of pure DPPC and second slightly shifted toward lower temperatures. These results may indicate the coexistence of various phases with different compositions. Changes to the heat flow might, therefore, be associated with formation of different domains consisting of different TBTC-DPPC compositions and consequently with different $T_{\mathrm{m}}$ values. MLVs formed from DMPC and from DMPE were used as model membranes. In case of

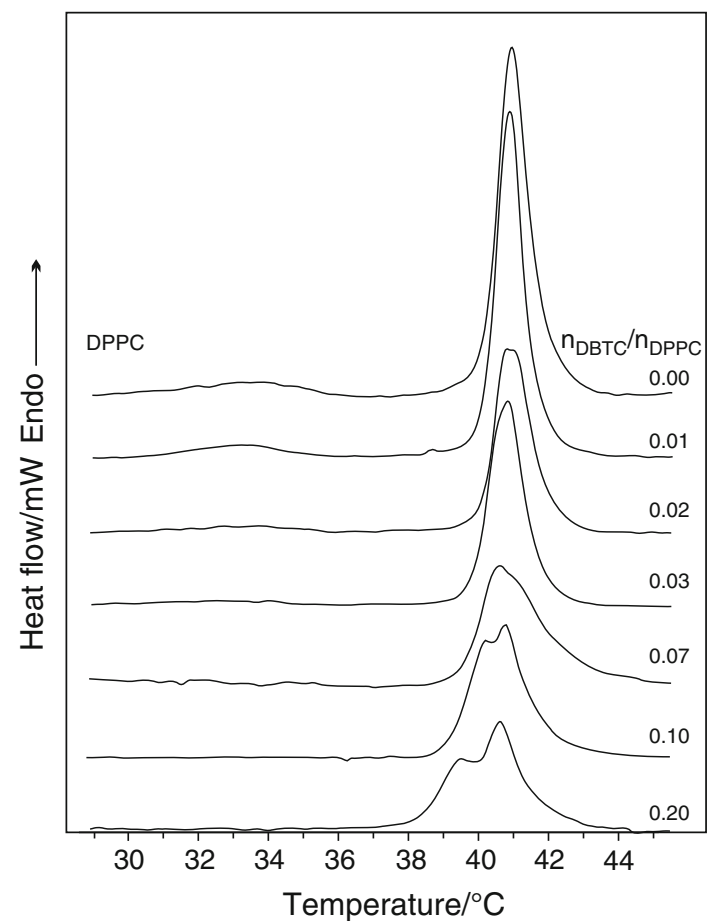

Fig. 2 DSC transition curves of MLV with DPPC modified with the DBTC

MLV formed from DMPC and DMPE, we observed similar effect as for DPPC lipids (Fig. 3).

In the case of MLV formed from DMPC, the presence of DBTC abolished pretransition, practically do not change the $T_{\mathrm{m}}$, decreased cooperativity and enthalpy of the main phase transition (Fig. 4), but do not separate the peak. In the case of MLV formed from DMPE, we observed that the main phase transition peak splits into two components for molar ratio 0.03 DMPE/DBTC. It is interesting that the second component moves toward higher temperatures with the increasing concentration of the compound. This suggests that DBTC disrupts the bilayer structure in DBTCdoped DPPC dispersion and probably a lamellar-nonlamellar phase transition appears.

Summarizing, these results suggest that DBTC interacts with the surface of the bilayers, probably partially locates at the surface of the lipid bilayer, and partially penetrates it.

The phase behavior and membrane fluidity changes induced by dibutyltin complex citrate were analyzed using probes: Laurdan, Prodan, and DPH. These fluorescent probes were used because each of them incorporates in a different region of a lipid bilayer. The DPH probes locate in the hydrophobic, while Laurdan and Prodan-in hydrophilic regions of the bilayer. Such differentiated incorporation of the probes gives insight into the structural changes caused by incorporation of investigated complex $[29,30]$. 


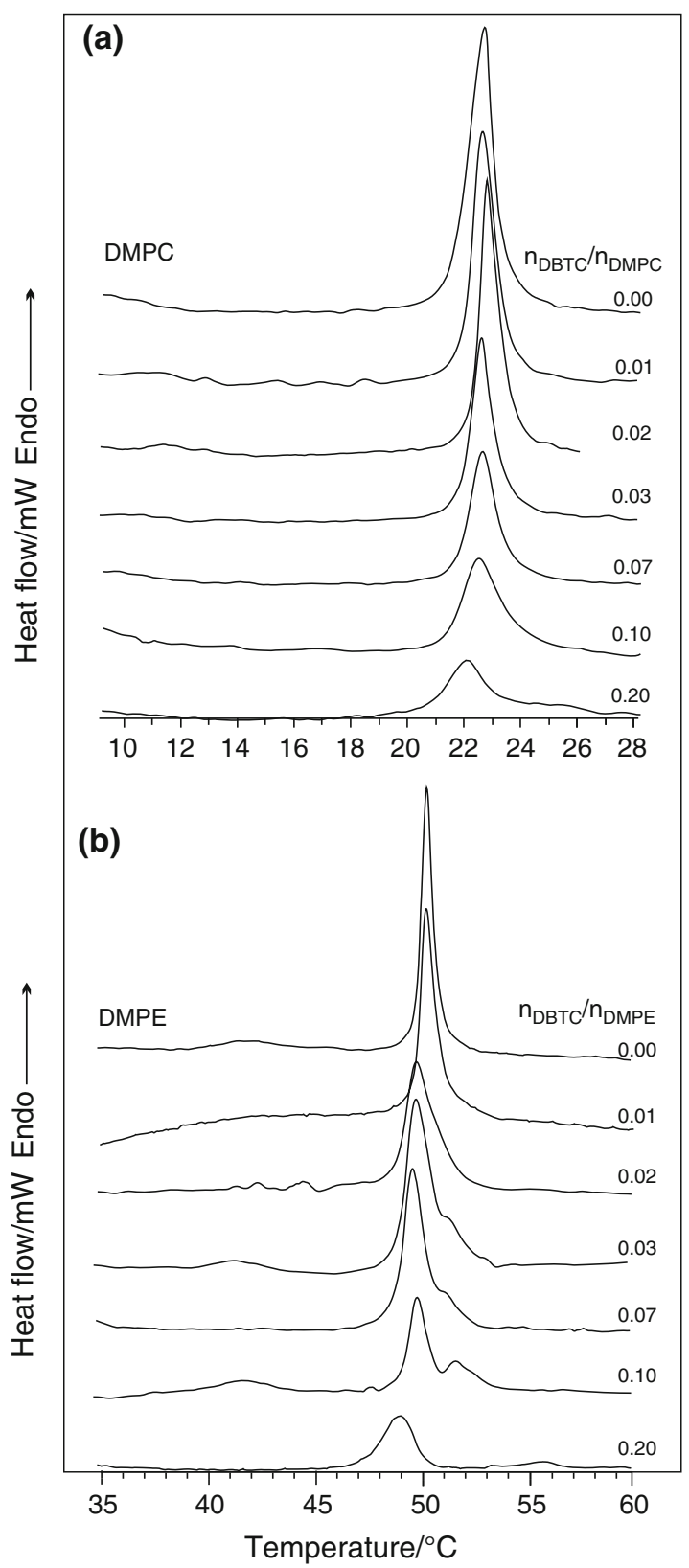

Fig. 3 DSC transition curves of MLV with DMPC (a) and with DMPE (b) modified with the DBTC

The effect of the DBTC on the fluidity and main phase transition of the MLV formed from DMPC was studied on the basis of anisotropy measured with the DPH probe. The fluorescence steady-state anisotropy is primarily related to the restriction of the rotational motion of the dye to the hydrocarbon chain packing order. Therefore, decrease of the anisotropy parameter can be explained by the structural perturbation of the bilayer hydrophobic region due to incorporation of studied compounds. The dependence of (a)
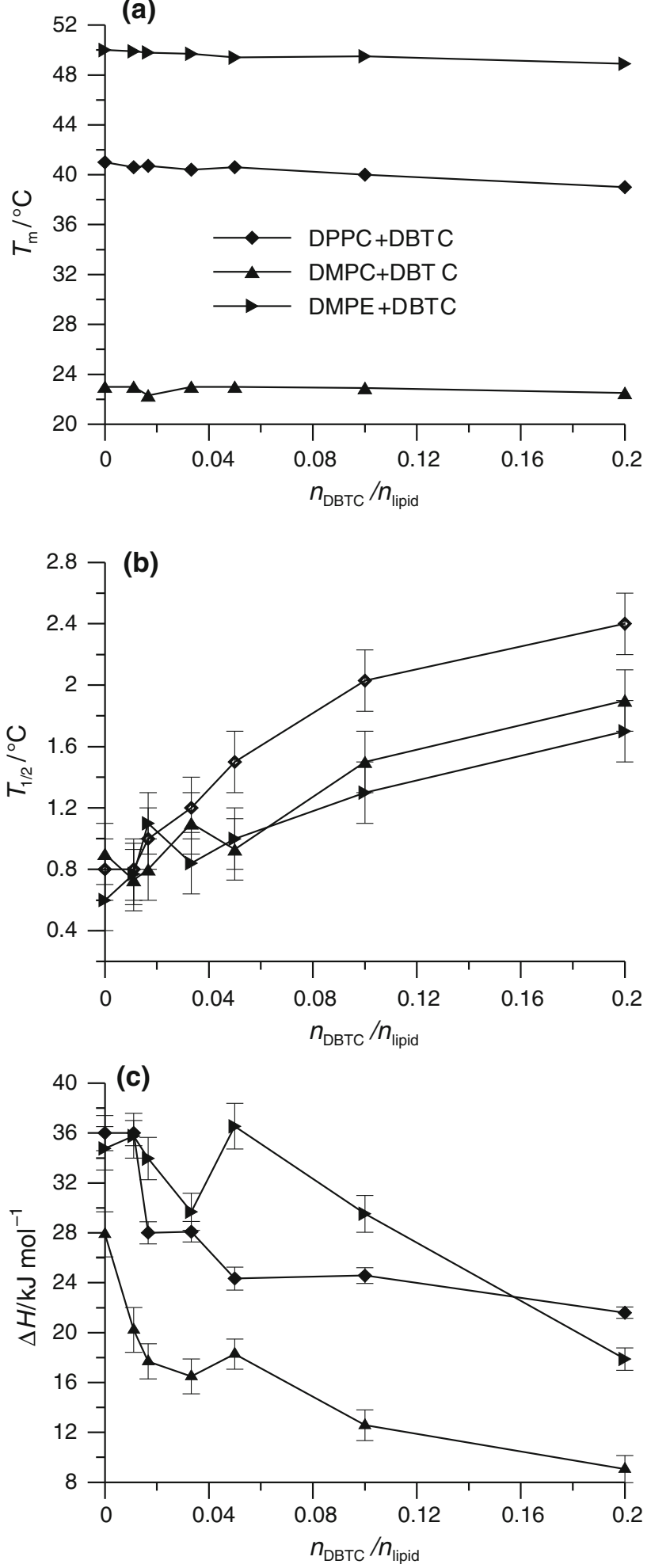

Fig. 4 Main phase transition temperatures $\left(T_{\mathrm{m}}\right)$ as a function of DBTC concentration (a), half width $\left(T_{1 / 2}\right)$ peak as a function of DBTC concentration (b), and enthalpies $(\Delta H)$ of the main phase transition as a function of DBTC concentration (c) 


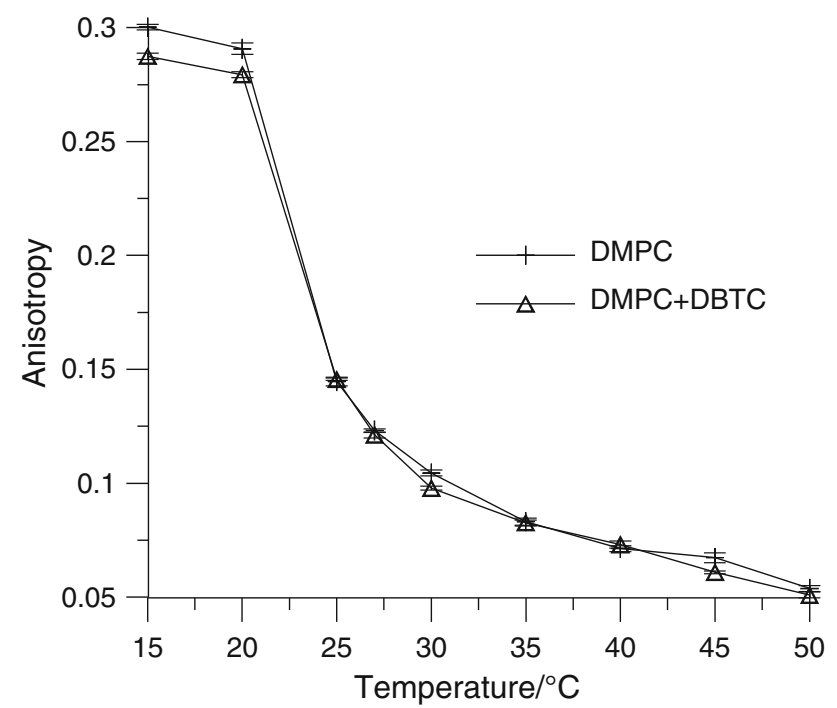

Fig. 5 Fluorescence anisotropy of DPH in MLV prepared from DMPC as a function of temperature

the DPH probe on temperature is presented in Fig. 5. The presence of DBTC practically does not change fluorescence anisotropy-we observed only slight decrease of the anisotropy of the gel-crystal phase without changing the $T_{\mathrm{m}}$. These results suggest that investigated compound had practically no influence on fluidity in the hydrophobic region of the lipid bilayers.

Using the Laurdan and Prodan probes, we monitored phospholipid phase transition, since spectral properties of both probes depend on the amount of water penetrating the appropriate regions of a bilayer. Laurdan is a membrane fluorescent probe that has the unique advantage of being sensitive to the phospholipid phase state. It is located in the hydrophilic-hydrophobic interface of the bilayer with the lauric acid tail anchored in the phospholipid acyl chain region [31]. The calculated values of general polarization (GP) of the Laurdan probe for MLV formed from DPPC are presented in Fig. 6a. DBTC slightly lowers the GP coefficient values both in the gel and liquid-crystalline phases, which indicates increasing disorder of the polar heads of lipid bilayer, but does not change temperature of the phase transition.

Comparing to Laurdan, Prodan has a different location in the lipid membrane, namely, closer to the aqueous phase. Such a location allows Prodan to be also sensitive to the pretransition occurring in the lipid polar head groups region [31]. Figure $6 \mathrm{~b}$ compares values of GP coefficients calculated on the basis of fluorescence intensity measured at different temperatures for the Prodan probe. For liposomes formed from DPPC, large changes in GP values are observed in the gel phase. The molar ratio of 0.07 DBTC/ lipid does not remove the pretransition totally but changes (a)

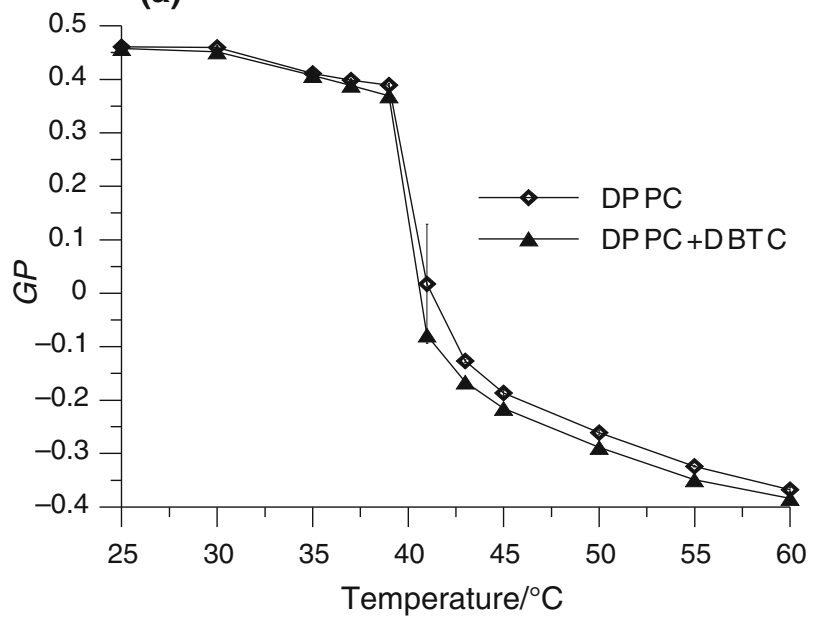

(b)

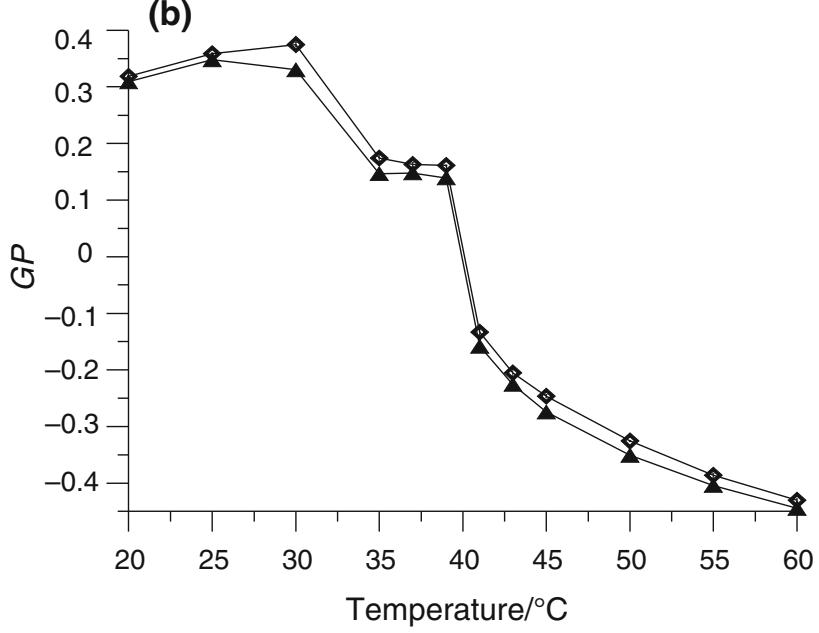

Fig. 6 Values of general polarization of Laurdan (a) and of Prodan (b) at different temperatures for DPPC liposomes with the tested DBTC (molar ratio DBTC/DPPC $=0.07)$

its temperature (Fig. 6b). These results suggest that DBTC strongly influences the gel state of the DPPC bilayer.

Summarizing these results confirm suggestions that DBTC interacts with lipid bilayer practically not influencing the fluidity of membranes and probably locates itself in the lipid head group region.

Interaction with plasmid DNA

The interaction of DBTC complexes with plasmid DNA was studied by using single-molecule fluorescence technique TCSPC-FCS. The main aim of the presented studies was to investigate ability and mode of binding for DBTC complexes with DNA molecule. In order to achieve a goal DNA molecules were labeled with two fluorescent dyes Hoechst $33258^{\circledR}$ and PicoGreen ${ }^{\circledR}$. Both dyes are commonly known as nucleic acid quantitation reagents. We use 


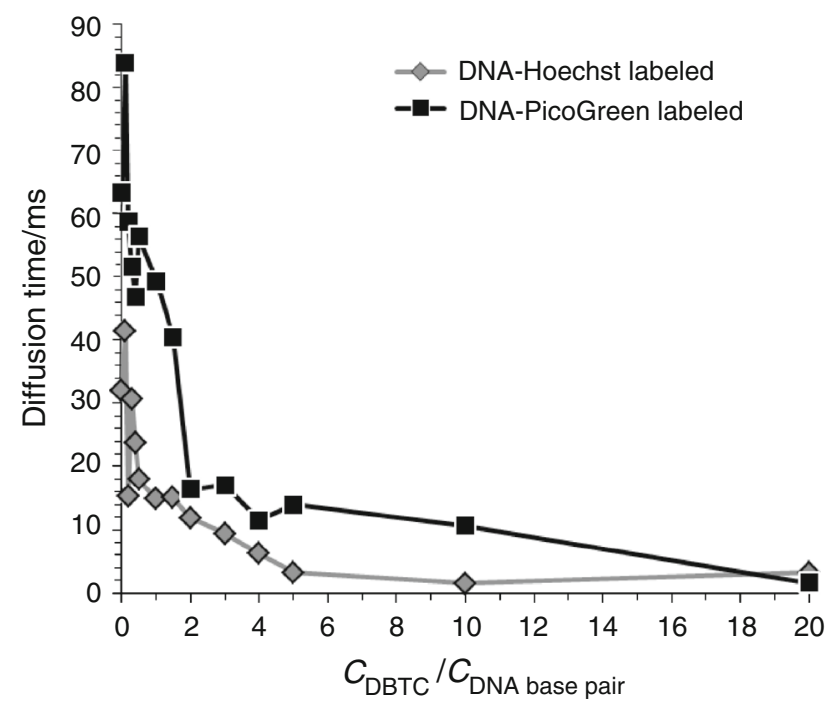

Fig. 7 Dependence of the diffusion time upon $C_{\mathrm{DBTC}} / C_{\mathrm{DNA}}$ base pair ratio in the solution. Dye/DNA base pair $=0.02$ both with Hoechst $33258^{\circledR}$ and PicoGreen ${ }^{\circledR}$ fluorophore

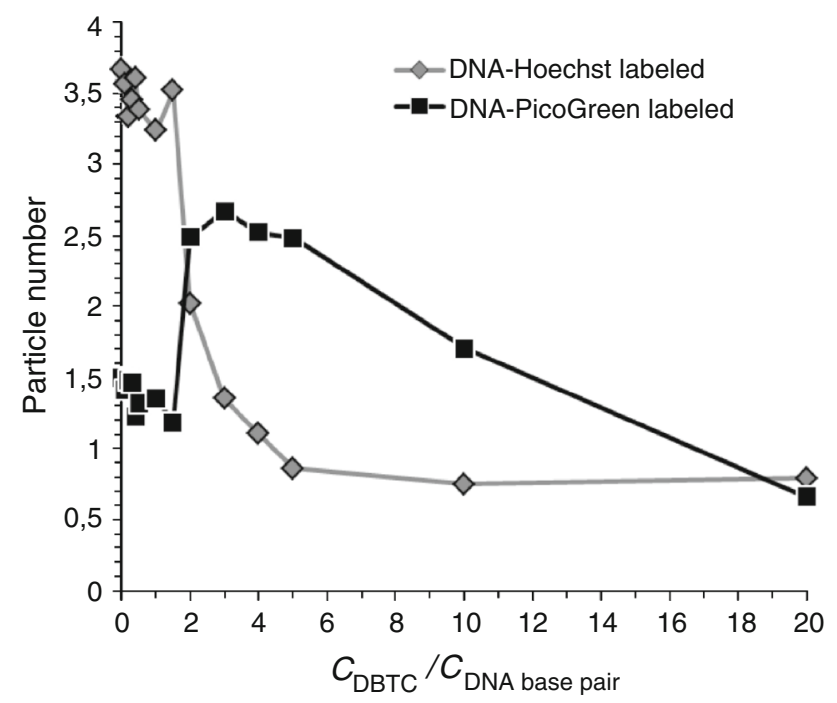

Fig. 8 Dependence of the particle number upon $C_{\mathrm{DBTC}} / C_{\mathrm{DNA}}$ base pair ratio in the solution. Dye/DNA base pair $=0.02$ both with Hoechst $33258^{\circledR}$ and PicoGreen ${ }^{\circledR}$ fluorophore

H33258, which displays sequence specificity, preferentially binds to the AATT stretch of DNA double helix [32-34] and exhibits a Stokes shift that heavily depends upon solvent polarity of the host environment [35]. It is reported that the interaction of H33258 may lead to DNA condensation at higher concentrations of the probe ([probe]:[DNA base]) 2.50:1 [36]. However, in our studies, the possibility of the DNA condensation by the probe molecules can be

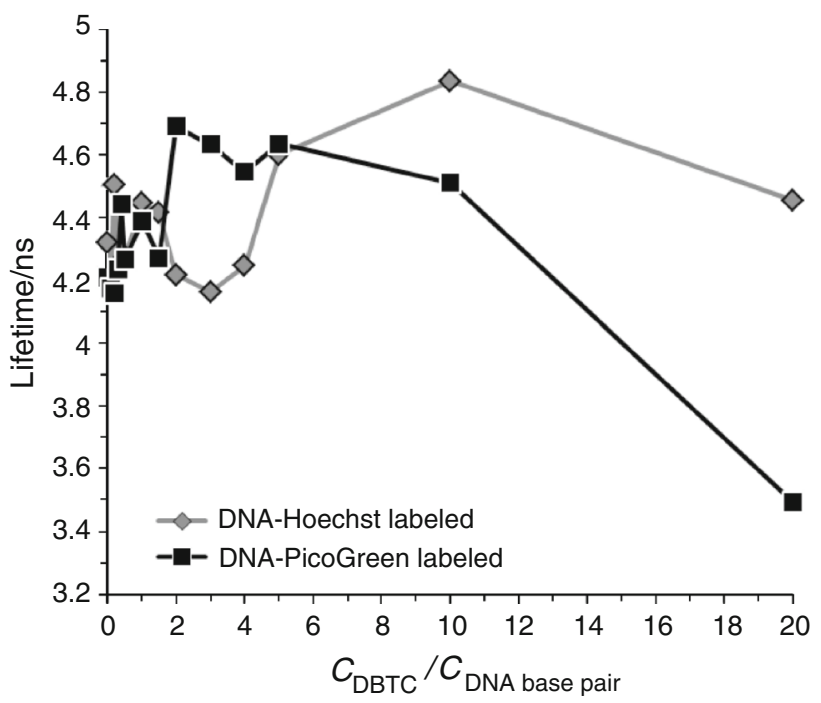

Fig. 9 Dependence of the lifetime upon $C_{\mathrm{DBTC}} / C_{\mathrm{DNA}}$ base pair ratio in the solution. Dye/DNA base pair $=0.02$ both with Hoechst $33258^{\circledR}$ and PicoGreen ${ }^{\circledR}$ fluorophore

ruled out as the samples contain ([probe]:[DNA base]) 1:50.

PicoGreen is a fluorescent probe that binds dsDNA and forms a highly luminescent complex when compared to the free dye in solution. In comparison to H33258, PicoGreen/ DNA complex is additionally stabilized by the interaction with the DNA phosphate group leading to an extremely strong binding constant [37].

The results of TCSPC-FCS measurements on the DNA with DBTC complexes are shown in Figs. 7, 8, and 9, as the dependences of diffusion time, particle number, and lifetime on DBTC/DNA $\mathrm{Dp}_{\mathrm{bp}}$ ratio, respectively.

In the absence of added DBTC complexes, the free uncoiled DNA has a diffusion time of 65 and $45 \mathrm{~ms}$ for PG and H33258 labeled, respectively (Fig. 7). This difference results from difference of the geometry of excitation and detection light in the confocal volume for PicoGreen and Hoechst dye. Within the supramolecular assembly with dibutyltin citrate, hydrodynamic radius of the DNA becomes smaller [38] and thereby as the concentration is increased the diffusion time of plasmid decreases continuously. The higher concentration of the DBTC in consequence gives a supramolecular assembly diffusing faster. At first we observed sevenfold decrease of the diffusion time for PG and fivefold decrease for Hoechst-labeled DNA-DBTC assemblies for the DBTC-to-base pair ratio below 2. Additionally, Figs. 7, 8, and 9 show that for the DBTC-to-base pair ratio below 2, diffusion time continuously decreases as well as PN $(1.5 \pm 0.2$ for PG/DNA and $3.5 \pm 0.2$ for Hoechst/DNA) and the lifetime remains stable within experimental error $(4.3 \pm 0.2 \mathrm{~ns}$ for both $\mathrm{PG} /$ 
DNA and Hoechst/DNA). Decrease of the diffusion time evidences that DBTC interacts with plasmid DNA molecule. DBTC-to-base pair ratio equal 2 seems to be threshold. In the DBTC-to-base pair ratio range from 2 to 5 , we observed further diffusion time decrease with simultaneous increase of the PN and lifetime for PGlabeled DBTC-DNA and simultaneous decrease of the PN and lifetime for Hoechst-labeled DBTC-DNA assemblies. For the DBTC-to-base pair ratio above 5 values of the PN about $0.8 \pm 0.2$ and diffusion time $=3.5 \pm 0.5$, Hoechstlabeled DNA reached minimum, whereas lifetime reached maximum equal to $4.8 \pm 0.2 \mathrm{~ns}$. In earlier studies revealing the compaction mechanism of $10 \mathrm{kbp}$ DNA, it was shown that the lifetime of $\mathrm{PG}$ is $4.3 \pm 0.2$ ns for free DNA and $3.3 \pm 0.2 \mathrm{~ns}$ for fully compacted DNA. For the DBTC complex interacting with PG-labeled DNA, we observed the same changes in the lifetime but for DBTC-to-base pair ratio fourfold higher than for Hoechst-labeled plasmid.

Fluorescence lifetime of dye molecules is a sensitive reporter on the local microenvironment. A change of the immediate microenvironment of the dye molecules forces a change of its lifetime and, inversely, the lifetime remains constant under unchanged conditions [35, 39]. This means that both dyes, PG and Hoechst do not "see" same environment. Hoechst 33258 binds in the minor groove, therefore, it should be more exposed to the media, whereas the PG molecule rigidly anchored between base pairs additionally stabilized by the interaction with the phosphate group of DNA is more competitive due to DBTC complex.

In summary, the experimental results for these three parameters (diffusion time, particle number, and lifetime) show that DBTC complex is able to interact and fully condense DNA.

For Hoechst-labeled DNA in the DBTC/DNA assembly the process is most efficient, taking place at the ratio $\mathrm{DBTC}_{\mathrm{DNA}} \mathrm{bp}_{\mathrm{bp}}=5$, i.e., the concentration of 5 dibutyltin citrate molecules per nucleotide.

The PG-labeled DNA binding results suggest the intercalative mode of binding for DBTC with DNA along with possible simultaneous electrostatic or chemical interactions between the $\mathrm{Sn}(\mathrm{IV})$ center and the phosphate backbone of the DNA helix.

\section{Conclusions}

In our study we investigated the interaction of the dibutyltin complex with citric acid with model membranes prepared from DPPC, DMPC, DMPE, and plasmid DNA.

We used calorimetric and fluorimetric methods to monitor changes in the gel phase to fluid lamellar phase transition of lipids. At low molar ratios, DBTC does not significantly change phase transitions of lipids. Transitions remain rather narrow and approximately symmetrical. With increasing concentration, the pretransition disappears and the main phase transition gets significantly broader and asymmetrical, with visible shoulders. A splitting of the main transition is observed at higher DBTC concentration. These results may indicate the coexistence of various phases of lipid bilayer with different compositions. The steady-state fluorimetry results show that DTAB practically does not change the fluidity of the lipid bilayer and slightly lowers the GP coefficient values both in the gel and liquidcrystalline phases. This leads to the conclusion that DBTC preferentially intercalated onto head group region to some extent into polar-apolar of the lipid bilayer.

The effect of DBCT on plasmid DNA was investigated using a single-molecule florescence technique and timecorrelated single photon counting fluorescence correlation spectroscopy (TCSPC-FCS). From the results we concluded that DBTC-DNA interaction occurs due to the interaction with the DNA phosphate group and charge neutralization which then leads to DNA compaction.

Acknowledgements The use of a DSC in the Institute of Agricultural Engineering of Wrocław University of Environmental and Life Sciences is gratefully acknowledged. Hanna Pruchnik acknowledges financial support of the statutory activities of the Department of Physics and Biophysics of the Wrocław University of Environmental and Life Sciences. Martin Hof acknowledges support from the Czech Science Foundation via Grant P208/12/G016 and the Praemium Academiae Award (Academy of Sciences of the Czech Republic). Teresa Kral acknowledges the MEYS ESF Project CZ.1.07/2.3.00/ 20.0092 .

Open Access This article is distributed under the terms of the Creative Commons Attribution License which permits any use, distribution, and reproduction in any medium, provided the original author(s) and the source are credited.

\section{References}

1. Crowe AJ. In: Fricker SP, editor. Metal complexes in cancer therapy. London: Chapman and Hall; 1994. p. 147-79.

2. Gielen M, Tiekink ERT. Tin compounds and their therapeutic potential. In: Gielen M, Tiekink ERT, editors. Metallotherapeutic drugs and metal-based diagnostic agents: the use of metals in medicine. Chichester: Wiley; 2005. p. 421-43.

3. Davies AD, Gielen M, Pannell KH, Tiekink ERT, editors. Tin chemistry: fundamentals, frontiers, and applications. New York: Wiley; 2008.

4. Pellerito L, Nagy L. Organotin(IV) ${ }^{n+}$ complexes formed with biologically active ligands: equilibrium and structural studies, and some biological aspects. Coord Chem Rev. 2002;224:111-50.

5. Dyląg M, Pruchnik H, Pruchnik F, Majkowska-Skrobek G, Ułaszewski S. Antifungal activity of organotin compounds with functionalized carboxylates evaluated by the micro-dilution bioassay in vitro. Med Mycol. 2010;48:373-83.

6. Hadjikakou SK, Hadjiliadis N. Antiproliferative and anti-tumor activity of organotin compounds. Coord Chem Rev. 2009;253:235-49. 
7. Pruchnik H, Lis T, Latocha M, Zielińska A, Ułaszewski S, Pelińska I, Pruchnik FP. Butyltin(IV) 2-sulfobenzoates: synthesis, structural characterization and their cytostatic and antibacterial activities. J Inorg Biochem. 2012;111:25-32.

8. Pruchnik H, Latocha M, Zielińska A, Ułaszewski S, Pruchnik FP. Butyltin (IV) 5-sulfosalicylates: structural characterization and their cytostatic activity. Polyhedron. 2013;49:223-33.

9. Casini A, Messori L, Orioli P, Gielen M, Kemmer M. Interactions of two cytotoxic organotin(IV) compounds with calf thymus DNA. J Inorg Biochem. 2001;85:297-300.

10. Prasad KS, Kumar LS, Chandan S, Jayalakshmi B, Revanasiddappa HD. Diorganotin(IV) complexes of biologically potent 4(3H)-quinazolinone derived Schiff bases: synthesis, spectroscopic characterization, DNA interaction studies and antimicrobial activity. Spectrochim Acta A. 2011;81:276-82.

11. Tariq M, Muhammad N, Sirajuddin M, Ali S, Shah NA, Khalid N, Tahir MN, Khan MR. Synthesis, spectroscopic characterization, $\mathrm{X}$-ray structures, biological screenings, DNA interaction study and catalytic activity of organotin(IV) 3-(4-flourophenyl)-2-methylacrylic acid derivatives. J Organomet Chem. 2013;723:79-89.

12. Nath M, Vats M, Roy P. Tri- and diorganotin(IV) complexes of biologically important orotic acid: synthesis, spectroscopic studies, in vitro anti-cancer, DNA fragmentation, enzyme assays and in vivo anti-inflammatory activities. Eur $\mathrm{J}$ Med Chem. 2013;59:310-21

13. Sirajuddin M, Ali S, Haider A, Shah NA, Shah A, Khan MR. Synthesis, characterization, biological screenings and interaction with calf thymus DNA as well as electrochemical studies of adducts formed by azomethine [2-((3,5 dimethylphenylimino)methyl)phenol] and organotin(IV) chlorides. Polyhedron. 2012;40:19-31.

14. de Carvalho Oliveira R, Santelli RE. Occurrence and chemical speciation analysis of organotin compounds in the environment: a review. Talanta. 2010;82:9-24.

15. Pruchnik H, Pruchnik FP. Butyltin(IV) citrates and tartrates: structural characterization and their interaction with nucleotides. J Organomet Chem. 2013;729:60-7.

16. Parasassi T, De Stasio G, Ravagnan G, Rusch RM, Gratton E. Quantitation of lipid phases in phospholipid vesicles by the generalized polarization of Laurdan fluorescence. Biophys J. 1991;60:179-89.

17. Lakowicz JR. Fluorescence polarization. In: Principles of fluorescence spectroscopy. New York: Plenum Press; 2006. p. $353-382$.

18. Ohtaka H, Kawasaki Y, Kodama M. Phase transitions of highly asymmetric chain-length $N$-lignocerylsphingomyelin (C24:0-SM) bilayer. J Therm Anal Calorim. 2013;113:1593-602.

19. Wu R-G, Wang Y-R, Wu F-G, Zhou H-W, Zhang X-H, Hou J-L. A DSC study of paeonol encapsulated liposomes, comparison the effect of cholesterol and stigmasterol on the thermotropic phase behavior of liposomes. J Therm Anal Calorim. 2012;109:311-6.

20. Gmajner D, Ulrih NP. Thermotropic phase behaviour of mixed liposomes of archaeal diether and conventional diester lipids. J Therm Anal Calorim. 2011;106:255-60.

21. Adjimatera N, Benda A, Blagbrough IS, Langner M, Hof M, Kral T. Fluorescence correlation spectroscopic studies of a single lipopolyamine-DNA nanoparticle. In: Berberan-Santos MN, editor. Fluorescence of supermolecules, polymers, and nanosystems. Berlin: Springer; 2008. p. 381-413.

22. Benda A, Hof M, Wahl M, Patting M, Erdmann R, Kapusta P. TCSPC upgrade of a confocal FCS microscope. Rev Sci Instrum. 2005;76:033106.

23. Kral T, Leblond J, Hof M, Scherman D, Herscovici J, Mignet N. Lipopolythiourea/DNA interaction: a biophysical study. Biophys Chem. 2010;148:68-73.
24. Magde D, Elson EL, Webb WW. Fluorescence correlation spectroscopy. II. An experimental realization. Biopolymers. 1974;13:29-61.

25. Humpolíčková J, Benda A, Sýkora J, Machán R, Kral T, Gasinska B, Enderlein J, Hof M. Equilibrium dynamics of spermineinduced plasmid DNA condensation revealed by fluorescence lifetime correlation spectroscopy. Biophys J. 2008;94:L17-9.

26. Adjimatera N, Kral T, Hof M, Blagbrough I. Lipopolyaminemediated single nanoparticle formation of calf thymus DNA analyzed by fluorescence correlation spectroscopy. Pharm Res. 2006;23:1564-73.

27. Winkler R, Keller S, Rädler J. Intramolecular dynamics of linear macromolecules by fluorescence correlation spectroscopy. Phys Rev E. 2006;73:41919.

28. Humpolíčková J, Stepánek M, Kral T, Benda A, Procházka K, Hof M. On mechanism of intermediate-sized circular DNA compaction mediated by spermine: contribution of fluorescence lifetime correlation spectroscopy. J Fluoresc. 2008;18:679-84.

29. Hendrich AB, Malon R, Pola A, Shirataki Y, Motohashi N, Michalak K. Differential interaction of Sophora isoflavonoids with lipid bilayers. Eur J Pharm Sci. 2002;16:201-8.

30. Dumas D, Muller S, Gouin F, Baros F, Viriot M-L, Stoltz JF. Membrane fluidity and oxygen diffusion in choresterol-enriched erythrocyte membrane. Arch Biochem Biophys. 1997;341:34-9.

31. Parasassi T, Krasnowska EK, Bagatolli L, Gratton E. Laurdan and prodan as polarity-sensitive fluorescent membrane probes. J Fluoresc. 1998;8:365-73.

32. Vega MC, Garcia Saez I, Aymami J, Eritja R, van der Marel GA, Van Boom JH, Rich A, Coll M. Three-dimensional crystal structure of the A-tract DNA dodecamer d(CGCAAATTTGCG) complexed with the minor-groove-binding drug Hoechst 33258. Eur J Biochem. 1994;222:721-6.

33. Parkinson JA, Ebrahimi SE, McKie JH, Douglas KT. Molecular design of DNA-directed ligands with specific interactions: solution NMR studies of the interaction of a m-Hydroxy Analog of Hoechst 33258 with d(CGCGAATTCGCG)2. Biochem. 1994;33: 8442-52.

34. Fornander LH, Wu L, Billeter M, Lincoln P, Nordén B. Minorgroove binding drugs: where is the second hoechst 33258 molecule? J Phys Chem B. 2013;117:2947-54.

35. Jin R, Breslauer KJ. Characterization of the minor groove environment in a drug-DNA complex: bisbenzimide bound to the poly[d(AT)]poly[d(AT)]duplex. Proc Natl Acad Sci USA. 1988;85:8939-42.

36. Saito M, Kobayashi M, Iwabuchi S, Morita Y, Takamura Y, Tamiya E. DNA condensation monitoring after interaction with Hoechst 33258 by atomic force microscopy and fluorescence spectroscopy. J Biochem. 2005;136(6):813-23.

37. Dragan AI, Casas-Finet JR, Bishop ES, Strouse RJ, Schenerman MA, Geddes CD. Characterization of PicoGreen interaction with dsDNA and the origin of its fluorescence enhancement upon binding. Biophys J. 2010;99(9):3010-9.

38. Sato YT, Hamada T, Kubo K, Yamada A, Kishida T, Mazda O. Folding transition into a loosely collapsed state in plasmid DNA as revealed by single-molecule observation. FEBS Lett. 2005;579:3095-9.

39. Schweitzer C, Scaiano JC. Selective binding and local photophysics of the fluorescent cyanine dye PicoGreen in doublestranded and single-stranded DNA. Phys Chem Chem Phys. 2003;5:4911-7. 